\title{
CONSTRUCTION OF HARMONIC MAPS BETWEEN PSEUDO-RIEMANNIAN SPHERES AND HYPERBOLIC SPACES
}

\author{
J. J. KONDERAK
}

(Communicated by Jonathan M. Rosenberg)

\begin{abstract}
Defined here is an orthogonal multiplication for vector spaces with indefinite nondegenerate scalar product. This is then used, via the Hopf construction, to obtain harmonic maps between pseudo-Riemannian spheres and hyperbolic spaces. Examples of harmonic maps are constructed using Clifford algebras.
\end{abstract}

\section{HARMONIC MAPS BETWEEN PSEUDO-RIEMANNIAN MANIFOLDS}

(I.1). In 1972 R. T. Smith ([S]) noticed that so-called orthogonal multiplications gave nice harmonic maps by applying the Hopf construction. This construction has not been done for vector spaces with an indefinite scalar product. There is a growing interest in physics in harmonic maps between pseudoRiemannian manifolds, especially since they have applications in string theory. For this reason it is useful to apply the Hopf construction to pseudo-Riemannian spheres and hyperbolic spaces to obtain new harmonic maps.

In Parts I and II we shall give a theoretical background for the construction of harmonic maps. Many of the properties shown for pseudo-Riemannian manifolds are transcriptions of those from the Riemannian case in that we follow the results of $[\mathrm{B}]$. For a review of the general properties of harmonic maps and the techniques used in this theory, see [EL1] and [EL2].

All the manifolds and maps considered in this paper are of the class $C^{\infty}$ unless otherwise specified.

I.2. Let $(M, g),(N, h)$ be pseudo-Riemannian manifolds and let $\phi: M \rightarrow$ $N$ be a map from $M$ to $N$; then one can construct a bundle of 1 -forms on $M$ with values in the pull-back bundle $\phi^{-1} T N$ :

$$
T^{*} M \otimes \phi^{-1}(T N) \text {. }
$$

This bundle is equipped with the connection $\nabla$ induced by the Levi-Civita connections on $T M$ and $T N$. The covariant derivative of the differential

Received by the editors April 17, 1989.

1980 Mathematics Subject Classification (1985 Revision). Primary 53C50; Secondary 58E20.

Key words and phrases. pseudo-Riemannian sphere and hyperbolic space, harmonic map, orthogonal multiplication, Clifford algebra.

This research was supported by the International Centre for Theoretical Physics, in Trieste, Italy. 
$\nabla d \phi$ is called the second fundamental form of $\phi$. It is said that $\phi$ is harmonic iff $\operatorname{Tr}_{g} \nabla d \phi=\sum_{a=1}^{m} \nabla d \phi\left(X_{a}, X_{a}\right)$ is equal to zero at each point of $M$, where $\left(X_{a}\right)_{1 \leq a \leq m}$ is a local orthonormal frame field on $M$. The section $\tau(\phi):=$ $\operatorname{Tr}_{g} \nabla d \bar{\phi}$ is called a tension field of $\phi$.

Example I.2.1. If $M=\mathbf{R}^{p, q}$ with $m=p+q$ and $\phi: \mathbf{R}^{p, q} \rightarrow \mathbf{R}$ then

$$
\tau(\phi)=-\sum_{a=1}^{p} \frac{\partial^{2} \phi}{\partial x_{a}^{2}}+\sum_{a=p+1}^{m} \frac{\partial^{2} \phi}{\partial x_{a}^{2}}
$$

where $x_{1}, \ldots, x_{m}$ are standard coordinates on $\mathbf{R}^{p, q}$. In this case the tension field is just a Laplacian of the function $\phi$. If $\phi: \mathbf{R}^{p, q} \rightarrow \mathbf{R}^{s, t}$ and $\phi=$ $\left(\phi_{1}, \ldots, \phi_{s+t}\right)$ with $\phi_{r}: \mathbf{R}^{p, q} \rightarrow \mathbf{R}, r=1, \ldots, m$, then $\tau(\phi)=\left(\tau\left(\phi_{1}\right), \ldots\right.$, $\left.\tau\left(\phi_{m}\right)\right)$.

Harmonic maps may be obtained as critical points of the energy functional for both the definite and indefinite case. For a given function $\phi$ between pseudo-Riemannian manifolds, the energy density of $\phi$ is defined as a function $e(\phi): M \rightarrow \mathbf{R}$ by

$$
e(x):=\frac{1}{2} \ll d_{x} \phi, d_{x} \phi \gg=\frac{1}{2} \sum_{a=1}^{m} h_{x}\left(d_{x} \phi\left(X_{a}\right), d_{x} \phi\left(X_{a}\right)\right)
$$

for each $x \in M$. Here $\ll, \gg$ denotes the Hilbert-Schmidt product on $\left[T^{*} M \otimes \phi^{-1}(T N)\right]_{x}$. Then, at least, locally the following integral

$$
E(\phi)=\int_{M} e(\phi)(x) \nu_{g}
$$

exists and is called the energy of $\phi$. Then a $C^{\infty}$ function is harmonic iff it is a critical point of the energy functional. Unlike the Riemannian case, the vanishing of the energy does not imply that the function $\phi$ is constant; the energy may even be negative.

I.3. Let $\varepsilon_{1}, \varepsilon_{2} \in\{-1,1\}$ and let $K_{1}, K_{2}$ be real positive numbers. Then we define

$$
\begin{aligned}
& \Sigma\left(\varepsilon_{1}, K_{1}\right):=\left\{x \in \mathbf{R}^{p, q} \mid\langle x, x\rangle=\varepsilon_{1} \cdot \frac{1}{K_{1}}\right\} \\
& \Xi\left(\varepsilon_{2}, K_{2}\right):=\left\{x \in \mathbf{R}^{s, t} \mid\langle y, y\rangle=\varepsilon_{2} \cdot \frac{1}{K_{2}}\right\}
\end{aligned}
$$

where $p+q=m$ and $s+t=n$ are integers. Here $\langle$,$\rangle denotes the scalar prod-$ ucts in the considered spacss. Then the space $\Sigma\left(\varepsilon_{1}, K_{1}\right)$ is a pseudo-Riemannian manifold of signature

$$
\begin{cases}(p-1, q) & \text { if } \varepsilon_{1}=-1 \\ (p, q-1) & \text { if } \varepsilon_{1}=1\end{cases}
$$

and has constant sectional curvature equal to $\varepsilon_{1} \cdot K_{1}$. The space $\Sigma\left(1, K_{1}\right)$ is called a pseudo-Riemannian sphere and $\Sigma\left(-1, K_{1}\right)$ is called a pseudo-Riemannian hyperbolic space. They will be denoted respectively by $\mathbf{S}^{p, q-1}$ and $\mathbf{H}^{p-1, q}$. 
By analogy we may describe geometrical properties of the space $\Xi\left(\varepsilon_{2}, K_{2}\right)$. In the present paper we construct harmonic maps between spaces of the above type.

There is the following natural homothetic transformation

$$
\chi_{1}: \Sigma\left(\varepsilon_{1}, K_{1}\right) \rightarrow \Sigma(\varepsilon, 1)
$$

such that $\chi_{1}(x)=\left(K_{1}\right)^{\frac{1}{2}} \cdot x$ where $x \in \mathbf{R}^{p, q}$. Then $\chi_{1}$ is an affine diffeomorphism. Similarly we construct this expression $\chi_{2}$ from $\Sigma\left(\varepsilon_{2}, K_{2}\right)$ to $\Sigma\left(\varepsilon_{2}, 1\right)$.

Remark I.3.1. It is easy to notice that if there are given functions $\phi$ and $\bar{\phi}$ such that the following diagram

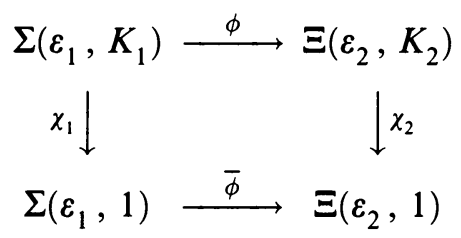

commutes, then $\phi$ is harmonic iff $\bar{\phi}$ is harmonic.

Because of the above remark, we shall concentrate only on the spaces of the sectional curvature \pm 1 . We shall also adopt the following notation: $\Sigma\left(\varepsilon_{1}\right):=$ $\Sigma\left(\varepsilon_{1}, 1\right)$ and $\Xi\left(\varepsilon_{2}\right):=\Xi\left(\varepsilon_{2}, 1\right)$.

Remark I.3.2. Let $A: \mathbf{R}^{p, q} \rightarrow \mathbf{R}^{q, p}$ be the map such that

$$
A\left(x_{1}, \ldots, x_{p}, x_{p+1}, \ldots, x_{p+q}\right)=\left(x_{p+1}, \ldots, x_{p+q}, x_{1}, \ldots, x_{p}\right) ;
$$

then $A$ is an affine diffeomorphism and $A\left(\Sigma\left(\varepsilon_{1}\right)\right)=\Sigma\left(-\varepsilon_{1}\right)$. It also has the following property: if $f: X \rightarrow \Sigma\left(\varepsilon_{1}\right)$ and $g: \Sigma\left(\varepsilon_{1}\right) \rightarrow Y$ are functions between pseudo-Riemannian manifolds, then $f$ is harmonic iff $A \circ f$ is harmonic; the same equivalence we have for $g$ and $g \circ A$. Of course, these properties are also valid for $\Xi\left(\varepsilon_{2}\right)$ and the respective affine diffeomorphism reversing the signature of the space $\mathbf{R}^{s, t}$.

Let $\mathbf{i}: \Sigma(\varepsilon) \rightarrow \mathbf{R}^{p, q}$ be an inclusion map, $\varepsilon \in\{-1,1\}$ and let $f: \mathbf{R}^{p, q} \rightarrow \mathbf{R}$ be a smooth map.

Lemma I.3.3. With respect to the above notations, we have that

$$
\tau(f \circ \mathbf{i})=\tau^{\mathbf{R}^{p, q}}(f)-\varepsilon(p+q-1) \frac{\partial f}{\partial \eta}-\varepsilon \frac{\partial^{2} f}{\partial \eta^{2}},
$$

where $\frac{\partial f}{\partial \eta}=d f\left(\frac{\partial}{\partial \eta}\right)$ and $\frac{\partial}{\partial \eta}$ is a vector field defined locally on a neighborhood of $\Sigma(\varepsilon)$ such that $\left\langle\frac{\partial}{\partial \eta}, \frac{\partial}{\partial \eta}\right\rangle=\varepsilon$ and $\frac{\partial}{\partial \eta}(x)$ is proportional to $x$.

Proof. We have a general formula for the tension field of a composition of functions:

$$
\tau(f \circ \mathbf{i})=d f(\tau(\mathbf{i}))+\operatorname{Tr}(\nabla d f)(d \mathbf{i}, d \mathbf{i})
$$


(cf. [EL1]). This formula is also valid for the pseudo-Riemannian case. On the other hand, we have that

$$
\tau^{\mathbf{R}^{p, q}}(f) \circ \mathbf{i}=\operatorname{Tr}(\nabla d f)(d \mathbf{i}, d \mathbf{i})+\varepsilon \frac{\partial^{2} f}{\partial \eta^{2}} .
$$

Let $\left(X_{a}\right)_{1 \leq a \leq m}$ denote an orthonormal basis on the tangent space $T_{x} \Sigma(\varepsilon)$ and let $\left(\gamma_{a}\right)_{1 \leq a \leq m}$ be the geodesics on $\Sigma(\varepsilon)$ such that $\dot{\gamma}_{a}(0)=X_{a}$ for $1 \leq a \leq m$. Let also $\xi_{a}=\left\langle X_{a}, X_{a}\right\rangle$. Then

$$
\begin{aligned}
\tau(\mathbf{i})(x) & =\sum_{a=1}^{m-1} \xi_{a}\left(\nabla_{\dot{\gamma}_{a}(0)}^{\mathbf{R}^{p, q}} \dot{\gamma}_{a}\right) \\
& =\sum_{a=1}^{m-1} \xi_{a} \cdot \gamma_{a}^{\prime \prime}(0) \\
& =-\varepsilon \sum_{a=1}^{m-1} \gamma_{a}(0) \\
& =-\varepsilon(p+q-1) \frac{\partial}{\partial \eta} .
\end{aligned}
$$

Hence we have that

$$
\tau(f \circ \mathbf{i})=\tau^{\mathbf{R}^{p, q}}(f)-\varepsilon(p+q-1) \frac{\partial f}{\partial \eta}-\varepsilon \frac{\partial^{2} f}{\partial \eta^{2}} .
$$

Observation I.3.4. If $\mathbf{w}$ is a $k$-homogeneous polynomial then

$$
\begin{aligned}
& \frac{\partial \mathbf{w}}{\partial \eta} \circ \mathbf{i}=k \cdot(\mathbf{w} \circ \mathbf{i}) \\
& \frac{\partial^{2} \mathbf{w}}{\partial \eta^{2}} \circ \mathbf{i}=k(k-1) \cdot(\mathbf{w} \circ \mathbf{i}) .
\end{aligned}
$$

Corollary 1.3.5. If $\mathbf{w}$ is a harmonic k-homogeneous polynomial on $\mathbf{R}^{p, q}$, then

$$
\tau(\mathbf{w} \circ \mathbf{i})=-\varepsilon k(p+q+k-2)(\mathbf{w} \circ \mathbf{i})
$$

hence $\mathbf{w}$ is an eigenfunction of the operator $\tau$.

We have the following property:

Lemma I.3.6. Let $\mathbf{j}: \Xi(\varepsilon) \rightarrow \mathbf{R}^{s, t}$ be a canonical inclusion and let $\psi: X \rightarrow \Xi(\varepsilon)$ be a smooth map between Riemannian manifolds; then the following conditions are equivalent:

(*) $\phi$ is harmonic

$(* *) \tau(\mathbf{j} \circ \phi)$ is orthogonal to $\Xi(\varepsilon)$ in $\mathbf{R}^{s, t}$.

For the proof see [S], [EL1]. The same lemma works for the pseudo-Riemannian case. 
Corollary I.3.7. If $\mathbf{w}: \mathbf{R}^{p, q} \rightarrow \mathbf{R}^{s, t}$ consists of $k$-homogeneous harmonic polynomials and $\mathbf{w}\left(\Sigma\left(\varepsilon_{1}\right)\right) \subset \Xi\left(\varepsilon_{2}\right)$, then

$$
\left.\mathbf{w}\right|_{\Sigma\left(\varepsilon_{1}\right)}: \Sigma\left(\varepsilon_{1}\right) \rightarrow \Xi\left(\varepsilon_{2}\right)
$$

is a harmonic map.

Proof. This corollary follows immediately from Corollary I.3.5 and Lemma I.3.6.

\section{ORTHOGONAL MULTIPLICATIONS}

II.1. Let $\Phi: \mathbf{R}^{p_{1}, q_{1}} \times \mathbf{R}^{p_{2}, q_{2}} \rightarrow \mathbf{R}^{s, t}$ be a bilinear map such that

$$
\langle\Phi(x, y), \Phi(x, y)\rangle=\langle x, x\rangle \cdot\langle y, y\rangle ;
$$

then $\Phi$ is called an orthogonal multiplication. Here $\langle$,$\rangle denotes the scalar$ products in respective vector spaces. For a given $\varepsilon_{1}, \varepsilon_{2} \in\{-1,1\}$, there are defined the following pseudo-Riemannian space forms.

$$
\begin{aligned}
& \Sigma\left(\varepsilon_{1}\right)=\left\{(x, y) \in \mathbf{R}^{p_{1}, q_{1}} \times \mathbf{R}^{p_{2}, q_{2}} \mid\langle x, x\rangle+\langle y, y\rangle=\varepsilon_{1}\right\} \\
& \Xi\left(\varepsilon_{2}\right)=\left\{(t, z) \in \mathbf{R} \times \mathbf{R}^{s, t} \mid t^{2}+\langle z, z\rangle=\varepsilon_{2}\right\} .
\end{aligned}
$$

Now we may apply the Hopf construction. Namely, the following map

$$
F: \mathbf{R}^{p_{1}, q_{1}} \times \mathbf{R}^{p_{2}, q_{2}} \rightarrow \mathbf{R} \times \mathbf{R}^{s, t}
$$

given by

$$
F(x, y)=(\langle x, x\rangle-\langle y, y\rangle, 2 \Phi(x, y))
$$

is a 2-homogeneous polynomial and

$$
F\left(\Sigma\left(\varepsilon_{1}\right)\right) \subset \Xi(1) .
$$

Moreover, if $p_{1}+q_{1}-p_{2}-q_{2}=0$, then $F$ is harmonic and hence, by Corollary I.3.7, we get that

$$
\left.F\right|_{\Sigma\left(\varepsilon_{1}\right)}: \Sigma\left(\varepsilon_{1}\right) \rightarrow \Xi\left(\varepsilon_{2}\right)
$$

is a harmonic map.

II.2. Several examples of orthogonal multiplications follow.

Example II.2.1. There is a natural orthogonal multiplication given by a tensor product

$$
\otimes: \mathbf{R}^{p_{1}, q_{1}} \times \mathbf{R}^{p_{2}, q_{2}} \rightarrow \mathbf{R}^{s, t}
$$

for all $\left(p_{1}, q_{1}\right),\left(p_{2}, q_{2}\right)$ and for $(s, t)$ induced by the first two signatures.

Example II.2.2. Let $Q_{1}: \mathbf{R} \rightarrow \mathbf{R}$ be a quadratic form such that $Q_{1}(t):=-t^{2}$. Then the Clifford algebra $C\left(Q_{1}\right)$ has a linear basis $\left\{1, e_{1}\right\}$ where $e_{1} \cdot e_{1}=1$. There is given a scalar product $\langle$,$\rangle on the space C\left(Q_{1}\right)$ such that

$$
\langle x, y\rangle:=\frac{1}{2}(x \cdot \bar{y}+y \cdot \bar{x})
$$


and with respect to this product, $C\left(Q_{1}\right)$ is isometric with $\mathbf{R}^{1,1}$. The multiplication in the Clifford algebra $C\left(Q_{1}\right)$ is orthogonal.

Example II.2.3. Let $Q_{2}: \mathbf{R}^{2} \rightarrow \mathbf{R}$ be a quadratic form such that $Q_{2}\left(t_{1}, t_{2}\right):=$ $-\left(t_{1}\right)^{2}-\left(t_{2}\right)^{2}$. Then the Clifford algebra $C\left(Q_{2}\right)$ has a linear basis $\left\{1, e_{1}, e_{2}\right.$, $\left.e_{1} \cdot e_{2}\right\}$ where $e_{1} \cdot e_{1}=1, e_{2} \cdot e_{2}=1$, and $e_{1} \cdot e_{2}=-e_{2} \cdot e_{1}$. It has a similarly defined scalar product as in the example above. Then $C\left(Q_{2}\right)$ is isometric with the space $\mathbf{R}^{2,2}$. The Clifford multiplication in $C\left(Q_{2}\right)$ is orthogonal.

Remark II.2.4. If we consider a quadratic form $\widetilde{Q}_{2}: \mathbf{R}^{2} \rightarrow \mathbf{R}$ such that $\widetilde{Q}_{2}\left(t_{1}, t_{2}\right)=-\left(t_{1}\right)^{2}+\left(t_{2}\right)^{2}$, then just as in Example II. 2.3 we obtain the Clifford algebra $C\left(\widetilde{Q}_{2}\right)$, which is vector space with a metric of signature $(2,2)$. The multiplication in $C\left(\widetilde{Q}_{2}\right)$ is orthogonal. This multiplication is equivalent with the one in $C\left(Q_{2}\right)$ in the sense of $[\mathrm{P}]$.

\section{Applications}

Using the properties proved in Parts I and II, we may obtain some new harmonic maps between pseudo-Riemannian manifolds.

Example III.1.1. The orthogonal multiplication from Example II.2.2 gives the following harmonic map:

$$
\phi_{1}: \mathbf{S}^{2,1} \rightarrow \mathbf{S}^{1,1}
$$

such that

$\phi_{1}\left(x_{1}, x_{2}, y_{1}, y_{2}\right)=\left(\left(x_{1}\right)^{2}-\left(x_{2}\right)^{2}-\left(y_{1}\right)^{2}+\left(y_{2}\right)^{2}, 2 x_{1} y_{1}-2 x_{2} y_{2}, 2 x_{1} y_{2}-2 x_{2} y_{1}\right)$, where $\left(x_{1}, x_{2}, y_{1}, y_{2}\right) \in C\left(Q_{1}\right)$. Using Remark I.3.2, we may obtain from $\phi_{1}$ another harmonic map, $\mathbf{H}^{1,2} \rightarrow \mathbf{S}^{1,1}$.

Example III.1.2. The orthogonal multiplication in $C\left(Q_{2}\right)$ from Example II.2.3 gives the following harmonics

$$
\phi_{2}: \mathbf{S}^{3,4} \rightarrow \mathbf{S}^{2,1},
$$

where $\phi_{2}\left(z_{1}, z_{2}\right)=\left(z_{1} \cdot \bar{z}_{1}-z_{2} \cdot \bar{z}_{2}, 2 z_{1} \cdot \bar{z}_{2}\right)$ for $\left(z_{1}, z_{2}\right) \in C\left(Q_{2}\right) \times C\left(Q_{2}\right)$. Applying Remark I.3.2 to $\phi_{2}$, we obtain another harmonic map:

$$
\begin{aligned}
\mathbf{S}^{4,3} & \rightarrow \mathbf{H}^{2,1} \\
\mathbf{S}^{3,4} & \rightarrow \mathbf{H}^{1,2} \\
\mathbf{H}^{4,3} & \rightarrow \mathbf{H}^{1,2} .
\end{aligned}
$$

As in the case of quaternions, our Clifford algebra $C\left(Q_{2}\right)$ máa be expressed as $C\left(Q_{1}\right) \times C\left(Q_{1}\right)$, with the product defined as follows,

$$
(x, y) \cdot(a, b)=(x a+y \bar{b}, x b+y \bar{a}),
$$

for $x, y, a, b \in C\left(Q_{1}\right)$. Using this property, we generalize a construction of R. T. Smith (cf. [S, Example 2.1.6]):

$$
\phi_{3}: C\left(Q_{2}\right) \times C\left(Q_{2}\right) \rightarrow \mathbf{R} \times C\left(Q_{1}\right) \times \mathbf{R} \times C\left(Q_{1}\right) \times C\left(Q_{2}\right)
$$


defined as

$$
\phi_{3}(x, y)=\left(x_{1} \bar{x}_{1}-x_{2} \bar{x}_{2}, 2 x_{1} \bar{x}_{2}, y_{1} \bar{y}_{1}-y_{2} \bar{y}_{2}, 2 y_{1} \bar{y}_{2}, \sqrt{2} x \cdot \bar{y}\right),
$$

where $x, y \in C\left(Q_{2}\right)$ and $x=\left(x_{1}, x_{2}\right), y=\left(y_{1}, y_{2}\right)$. Then $\phi_{3}$ is a 2homogeneous harmonic polynomial and $\phi_{3}\left(\mathbf{S}^{4,3}\right) \subset \mathbf{S}^{4,5}$. Hence by Corollary I.3.7 and Remark I.3.2, $\phi_{3}$ gives us the following harmonic maps:

$$
\begin{gathered}
\mathbf{S}^{4,3} \rightarrow \mathbf{S}^{4,5} \\
\mathbf{H}^{3,4} \rightarrow \mathbf{S}^{4,5} \\
\mathbf{S}^{4,3} \rightarrow \mathbf{H}^{4,5} \\
\mathbf{H}^{3,4} \rightarrow \mathbf{H}^{5,4} .
\end{gathered}
$$

Example III.1.4. R. T. Smith applied the Veronese map to construct harmonic maps between spheres (cf. [S]). The same construction works in our pseudoRiemannian case. Namely, if $x, y, z$ belong to $C\left(Q_{1}\right)$ or to $C\left(Q_{2}\right)$, then the following map,

$$
(x, y, z) \rightarrow \sqrt{3}\left(x \bar{y}, x \bar{z}, y \bar{z}, \frac{1}{2}(x \bar{x}-y \bar{y}), \frac{1}{2 \sqrt{3}}(x \bar{x}+y \bar{y}-2 z \bar{z})\right),
$$

is 2-homogeneous and harmonic and preserves respective pseudo-Riemannian spheres. Hence, using once again Corollary I.3.7 and Remark I.3.2, we obtain from the above function four examples of harmonic maps.

Example III.1.5. Here is another pseudo-Riemannian variant of the Smith construction: if $x, y$ belongs to $C\left(Q_{1}\right)$ or to $C\left(Q_{2}\right)$, then the following map,

$$
(x, y) \rightarrow\left(x^{2}, y^{2}, \sqrt{2} x \bar{y}\right),
$$

is 2-homogeneous and harmonic and preserves respective pseudo-Riemannian spheres. Hence it produces examples of harmonic maps.

Remark III.1.6. Other harmonic homogeneous polynomials preserving pseudoRiemannian spheres may be obtained from Toth's classification theorem (cf. [T]), replacing real numbers by elements of $C\left(Q_{1}\right)$ or $C\left(Q_{2}\right)$.

Final remark. In many constructions we use conjugations of elements of Clifford algebras $C\left(Q_{1}\right)$ and $C\left(Q_{2}\right)$ in the formulas defining harmonic homogeneous polynomials. In some cases, even without the conjugations, these polynomials define desired maps. However, with these conjugations our harmonic maps have nice symmetries. Our maps are invariant with respect to the action of the Lie groups of elements whose scalar product is \pm 1 in $C\left(Q_{1}\right)$ or $C\left(Q_{2}\right)$. The manifolds obtained by the standard factorization have natural pseudo-Riemannian structures. We may also factorize our harmonic maps. In each of the cases we obtain new harmonic maps. They may be regarded as a variant of the harmonic maps between projective spaces in the Riemannian case. 


\section{REFERENCES}

[B] P. Baird, Harmonic maps with symmetries, harmonic morphisms, and deformation of metrics, Res. Notes in Math., no. 87, Pitman, Boston, MA, 1983.

[EL1] J. Eells and L. Lemaire, Selected topics in harmonic maps, CBMS Regional Conf. Series in Math., no. 50, Conf. Board Math. Sci., Washington, D. C., 1983.

[EL2] _ Another report on harmonic maps, Bull. London Math. Soc. (to appear).

[P] M. Parker, Orthogonal multiplications in small dimensions, Bull. London Math. Soc. 15 (1983), 368-372.

[S] R. T. Smith, Harmonic maps of spheres, thesis, Warwick University, 1972.

[T] G. Toth, Classification of quadratic harmonic maps of $S^{3}$ into spheres, Indiana Univ. Math. J. 30 (1987), 231-239.

International Centre for Theoretical Physics, P. O. Box 586, Miramare, Strada COSTIERA 11, 34100 Trieste, ItALY

Current address: Dipartimento di Matematica, Facoltà Scienze, Università di Salerno, 84100 Salerno, Italy 